27th EPS Conference on Contr. Fusion and Plasma Phys. Budapest, 12-16 June 2000 ECA Vol. 24B (2000) 1144-1147

\title{
ANOMALOUS PARTICLE CONVECTION IN TOKAMAKS
}

\author{
Satish Puri \\ Max-Planck-Institut für Plasmaphysik, EURATOM Association, 85748 Garching
}

Radial particle convection rather than Ware pinch is the correct Onsager symmetry counterpart of bootstrap current. Coupled with enhanced radiative collisionality [1], it is possible to account for diverse tokamak-transport anomalies including (i) inward particle convection, (ii) anomalous current penetration, (iii) self-acceleration of plasmas during Ohmic $\mathrm{H}$-mode, counter to the expected $\mathbf{E} \times \mathbf{B}$ direction, (iv) enhanced electron thermal conduction, (v) magnetic field, density and isotope mass dependence, as well as (vi) the origin of linear (LOC) and saturated (SOC) Ohmic confinement regimes. Insight is offered into (vii) physics of $\mathrm{L}-\mathrm{H}$ transitions, and (viii) power degradation in auxiliary heated plasmas. The possibilty of using (ix) rotation-induced inward particle convection for plasma refueling is discussed.

Engelhardt's [2] conjecture of anomalous inward particle convection in tokamaks has proved to be an enduring enigma of tokamak transport physics. In this paper it is shown that radial particle convection is the Onsager symmetry counterpart of bootstrap current. Radially outward flux of trapped particles releases mechanical toroidal momentum to circulating electrons and ions giving rise to bootstrap current. Conversely, the momentum imparted to the trapped particles by collisions with the toroidally drifting circulating particles results in radial convection. Ware pinch [3], on the other hand, requiring neither collisions nor current for its existence, occurs even for a single trapped particle in the presence of a toroidal electric field. Ware pinch is often incorrectly identified as the Onsager symmetry complement to bootstrap current.

Generalizing Wesson's [4] derivation of Ware pinch, the toroidal equation of motion in a tokamak may be written as

$$
\frac{d}{d t}\left(m_{j} v_{\phi}\right)=e_{j}(\mathbf{E}+\mathbf{v} \times \mathbf{B})_{\phi}+F_{\phi}
$$

where the force $F_{\phi}$ is of non-electromagnetic origin. For trapped particles the integral between the bounces of the term on the left-hand side vanishes and on the average

$$
<(\mathbf{v} \times \mathbf{B})_{\phi}>=-E_{\phi}-F_{\phi} / e_{j} .
$$

Since

$$
(\mathbf{v} \times \mathbf{B})_{\phi}=v_{r} B_{\theta},
$$

the time-averaged pinch velocity for trapped particles is given by

$$
<v_{r}>=-\frac{E_{\phi}}{B_{\theta}}-\frac{F_{\phi}}{e_{j} B_{\theta}} .
$$

The same result may be derived using the conservation of canonical angular momentum in an axisymmetric geometry. The first term on the right-hand side of (4) is the Ware pinch. The second term represents additional pinch due to non-electromagnetic forces. 
27th EPS CCFPF 2000; S. Puri: Anomalous Particle Convection in Tokamaks

Apart from a precessional motion, trapped particles in a tokamak are unable to drift toroidally due to the conservation of the longitudinal adiabatic invariant. Averaged over the trapped population, the net precessional drift is small and plays no significant role. For all practical purposes the trapped particles may be considered toroidally stationary. The force due to the toroidally drifting circulating particles colliding against the stationary trapped particles plays the role of the non-electromagnetic force $F_{\phi}$. This gives rise to radially inward particle convection. Conversely, collisional processes causing trapped-particle diffusion exert an oppositely directed toroidal force giving rise to bootstrap current. Hence bootstrap current and radial convection are Onsager symmetric complementary phenomena involving only the non-electromagnetic force term $F_{\phi}$. Ware pinch, on the other hand, involves only the electromagnetic force term $e_{j} E_{\phi}$ which has no counterpart in bootstrap current drive. Reversing the direction of either $E_{\phi}$ or $B_{\theta}$ would change Ware pinch to an anti-pinch, which is the correct Onsager symmetry complement of Ware pinch involving only the electromagnetic force $e_{j} E_{\phi}$.

The force exerted on the trapped electrons and ions through collisions against circulating electrons and ions is given by

$$
F_{\phi e}=\nu_{e e} m_{e} n_{e} v_{\phi e}+\nu_{i e} m_{i} n_{i} v_{\phi i},
$$

and

$$
F_{\phi i}=\nu_{e i} m_{e} n_{e} v_{\phi e}+\nu_{i i} m_{i} n_{i} v_{\phi i},
$$

causing an effective banana regime radial convection given by

$$
v_{r e}^{\text {banana }}=\frac{\varepsilon^{1 / 2}\left(\nu_{e e} m_{e} n_{e} v_{\phi e}+\nu_{i e} m_{i} n_{i} v_{\phi i}\right)}{e B_{\theta}}
$$

and

$$
v_{r i}^{b a n a n a}=-\frac{\varepsilon^{1 / 2}\left(\nu_{e i} m_{e} n_{e} v_{\phi e}+\nu_{i i} m_{i} n_{i} v_{\phi i}\right)}{e B_{\theta}} .
$$

Radial convection continues undiminished in the plateau regime as

$$
v_{r e}^{\text {plateau }}=\frac{\varepsilon^{2} v_{t e}\left(\nu_{e e} m_{e} n_{e} v_{\phi e}+\nu_{i e} m_{i} n_{i} v_{\phi i}\right)}{q R \nu_{e e} e B_{\theta}}
$$

and

$$
v_{r i}^{\text {plateau }}=-\frac{\varepsilon^{2} v_{t i}\left(\nu_{e i} m_{e} n_{e} v_{\phi e}+\nu_{i i} m_{i} n_{i} v_{\phi i}\right)}{q R \nu_{i i} e B_{\theta}} .
$$

This contrasts with Ware pinch which steadily diminishes with increasing collisionality in the plateau regime. As one moves from the plateau regime to the Pfirsch-Schlüter regime, radial convection ceases suddenly. As will be shown later, this abrupt cessation/inception of radial convection can precipitate L- $\mathrm{H}$ transitions.

Since rotations always accompany radial-force-balance requirements in a plasma, pinches are of universal occurence. For example, during Ohmic current drive, the current-carrying circulating electrons move with the average toroidal velocity $v_{\phi e}=$ $-\sigma E_{\phi} / e n_{e}$; whereas the trapped electrons have zero average toroidal speed, giving rise to the neoclassical correction to Spitzer resistivity. The collisional force $F_{\phi}=\nu_{e e} m_{e} v_{\phi e}$ on the trapped electrons causes an inward pinch of velocity

$$
v_{i n}=-\frac{F_{\phi e}}{e B_{\theta}} \approx \frac{\nu_{e e} m_{e} \sigma}{e^{2} n_{e}} \frac{E_{\phi}}{B_{\theta}} \approx \frac{\nu_{e e}}{\nu_{e i}} \frac{E_{\phi}}{B_{\theta}} .
$$


27th EPS CCFPF 2000; S. Puri: Anomalous Particle Convection in Tokamaks

The radial pinch in (11) is in addition to the Ware pinch $v_{i n}^{\text {Ware }}=E_{\phi} / B_{\theta}$; it will exceed Ware pinch by a large factor for the case of enhanced electron-electron collisionality due to Kirchhoff thermal radiation [1]. The particle pinches lead to steepening of density profiles until a balance is reached with plasma diffusion and the particle sources. Since radial inward convection absorbs precisely the amount of canonical momentum that the bootstrap current releases, there is no free (without external injection) bootstrap current. The only free bootstrap current comes from direct neutral-particle injection (e.g., pellets or neutral beams) into the plasma interior. A small remnant of bootstrap current persists even in the Pfirsch-Schlüter regime.

Although implicit in all neoclassical treatments that include conservation of canonical angular momentum, radial particle convection finds neither explicit mention nor recognition in existing literature; neoclassical theory as currently practiced is fundamentally flawed.

Coupled with enhanced $\nu_{e e}$, radial convection leads to enhanced perpendicular viscosity, resulting in the observed anomalous current penetration in Ohmically heated tokamak plasmas. In steady state, the convected momentum $\left(\nu_{e e} / \nu_{e i}\right) m_{e} n_{e} v_{\phi e}$ lost per collision interval $\nu_{e i}^{-1}$ is redeposited via particle diffusion resulting in an effective perpendicular viscosity

$$
\eta_{\perp e}=m_{e} n_{e} \chi_{e}
$$

where $\chi_{e}$ is the neoclassical electron thermal diffusivity. The viscous current diffusion time $a^{2} / \chi_{e}$ given by (12) is close to the experimentally observed current penetration time and is orders of magnitude less than the resistive penetration time $\mu_{0} \sigma a^{2}$. The enhanced perpendicular viscosity would allow steady-state tokamak operation using only shallow (at $r / a \sim 0.67$ ) current drive near the plasma periphery. Efficient $\left(\gamma_{\text {normalized }} \gtrsim 2 A m^{-2} / W\right)$ steady-state tokamak operation would be possible using Alfven-wave current drive and without reliance on the non-existent, free bootstrap current. The high efficiency of Alfven-wave current drive is due to the low energy requirement for low-phase-velocity waves [5]. Although the wave energy of low-phase-velocity waves is imparted primarily to trapped electrons, in a plasma with radiatively enhanced $\nu_{e e}[1]$, the momentum imparted to the trapped electrons is redistributed among the entire electron population [6] before it is lost to the ions. This results in a current-drive efficiency consistent with the findings of Ref.[5].

Toroidal electron and ion drifts are inherent to a tokamak plasma. A moderate warming of the electron component near the edge can shift the electrons at the plasma periphery from the Pfirsch-Schlüter to the plateau regime initiating an abrupt inward electron pinch. The electron pinch imparts a co-directed momentum to the plasma near the edge. The co-rotating ions, in turn, cause inward ion convection, thereby precipitating an L-H transition. Thus, warming the edge electrons in the Pfirsch-Schlüter regime is one possible mechanism for triggering $\mathrm{L}-\mathrm{H}$ transitions. The spontaneous co-directed plasma spin-up without any external injection of toroidal momentum would explain the self-acceleration of a tokamak plasma during Ohmic $\mathrm{H}$-mode seen in Alcator C-Mod [7]. The fact that the co-directed spin-up is in a direction contrary to the $\mathbf{E} \times \mathbf{B}$ drift is also consistent with experimental observations [7]. The pinch process was initially discovered [8] in a situation resembling $\mathrm{L}-\mathrm{H}$ transitions.

Ambipolarity is violated during the transient L- $\mathrm{H}$ transition phase and is balanced by toroidal spin-up. Neoclassical transport is not necessarily ambipolar; ambipolarity is conserved only under the constraint that no net toroidal momentum is set free. 
27th EPS CCFPF 2000; S. Puri: Anomalous Particle Convection in Tokamaks

Inward radial convection opens up the possibility of tokamak refueling using the combination of inexpensive, low-energy, shallow neutral-beam injection for the particle and ion-momentum source and shallow Alfven-wave current drive as the electron momentum source. In principle, it would be possible to produce an extremely clean plasma because of the reduced dependence on wall-contaminated gas acting as the particle source. A detailed study of this possibility is now in progress.

The remaining transport anomalies listed at the start of this paper can be resolved via enhanced radiative collisionality contributed by Kirchhoff thermal radiation $[1,9]$. The Kirchhoff law approach has the critical advantage that it requires the knowledge of only the one-partcle distribution function without any need of expansion schemes. The expansion schemes in the statistical-theory approaches result in compromises that severely curtail their domain of applicability. This is clearly seen in Klimontovich's [10] derivation of the Balescu-Lenard equation as explained in the following paragraph.

Solubility is exacted by discarding the first term in Eq.(10.7) of Ref.[10] using the argument that since spectral functions relax faster than the distribution function, the time-dependent term is small compared to the other terms. This would indeed be true if $\omega \ll \nu / \epsilon$, where $\epsilon=1 /\left(n_{e} \lambda_{D}^{3}\right)$ is the plasma parameter. But for the highharmonic Bernstein waves with $\omega \sim 10^{13} \mathrm{~s}^{-1} \gg \nu / \epsilon$, since the time derivative $\partial / \partial t$ effectively multiplies the first term in Eq.(10.7) by $\omega$, rather than becoming negligibly small, as assumed by Klimontovich and others, the first term dominates Eq.(10.7), notwithstanding the fast relaxation of the spectral functions. The expansion procedure in powers of $\epsilon$ breaks down, and the validity of the Balescu-Lenard approach is vitiated. Hence, the thermodynamically derived Kirchhoff law approach is currently the only recourse available for estimating radiative collisionality.

In summary, it is possible to resolve the principal anomalies of tokamak transport within the framework of neoclassical theory supplemented by (i) rotation-driven particle pinches, and (ii) enhanced radiative collsionality. This supplemented version of neoclassical theory will be referred to as supraclassical theory. The agreement of the theoretically derived scaling laws with experimental results over an extremely wide range of plasma parameters implies that supraclassical transport is the dominant transport mechanism in tokamak plasmas.

\section{REFERENCES}

[1] S. Puri, Phys. Plasmas 5 (1998) 2932.

[2] K. Behringer, W. Engelhardt, W. Feneberg, G. Fussmann, Bull. APS, Vol.25, Number 8 (1980) 875.

[3] A. A. Ware, Phys. Rev. Lett. 25 (1970) 15.

[4] J. Wesson, in Tokamaks, Clarendon, Oxford (1997), p.164.

[5] D. J. H. Wort, Plasma. Phys. 13 (1971) 258.

[6] Y. Kolesnischenko, V. Parail, and G. Pereverzev, in Reviews of Plasma Physics, Consultants Bureau, New York (1992), Vol. 17.

[7] I. H. Hutchinson, J. E. Rice, R. S. Granetz, and J. A. Snipes, Phys. Rev. Lett. 84 (2000) 3330.

[8] S. Puri, Plasma Phys. Control. Fusion 41 (1999) L35.

[9] S. Puri, Proc. 25th EPS, Prague (1998), Paper P4.202.

[10] Yu. L. Klimontovich, in The Statistical Theory of Non-Equilibrium Plasmas, Pergamon, Oxford (1967), pp. 117-120. 